\title{
COVID-19: a global pandemic causing local threat in Nepal
} Janak Koirala ${ }^{1 *}$, Sangita Basnet ${ }^{2}$

\author{
'Professor Emeritus, Department of Medicine, Division of Infectious Diseases \\ Southern Illinois University School of Medicine, Springfield, Illinois, USA \\ ${ }^{2}$ Professor, Pediatrics and Critical Care Medicine \\ Southern Illinois University School of Medicine, Springfield, Illinois, USA
}

Keywords: COVID-19, coronavirus, SARS CoV-2,

Nepal

Note: This is an "invited Editorial", written by the authors upon request of Professor Umid Kumar Shrestha, MD, PhD, FACG, Editor-in-Chief of Journal of Advances in Internal Medicine, under a special circumstance of COVID-19 pandemic causing local threat in Nepal.

This work is licensed under a Creative Commons Attribution 4.0

Unported License.

\section{Editorial:}

Coronavirus, first described in 1966, is known to cause mild respiratory illnesses in humans. However, more virulent form of coronaviruses causing serious respiratory illnesses were identified in the past 2 decades. SARS-CoV (coronavirus) caused outbreaks of severe acute respiratory syndrome (SARS) in 29 countries between 2002-2004. MERS-CoV, which was first identified in 2012, caused Middle East respiratory syndrome (MERS) with high mortality. The current pandemic Coronavirus Disease 2019 (COVID-19) was identified in December 2019 after a cluster of patients with pneumonia of unknown etiology leading to ARDS (Acute Respiratory Distress Syndrome) were admitted in Hubei Province of China. It was subsequently linked to the seafood and wet animal market of Wuhan, China. ${ }^{1,2}$

The etiology of COVID-19 has been identified as a novel coronavirus, SARS-CoV-2, which belongs to the B lineage of beta-coronaviruses. ${ }^{3}$ This novel coronavirus is closely related to SARS CoV-1 that caused an epidemic in 2003-2004, and its genome is $96 \%$ identical to bat coronavirus. ${ }^{4}$ SARS-CoV-2 appears to have a receptor-binding domain (RBD) in its spike protein that binds with high affinity to Angiotensin-Converting Enzyme-2 (ACE-2) receptors in human cells. These receptors are present in the lungs, heart, kidneys, and gastrointestinal tract where they facilitate viral entry. ${ }^{5}$ The RBD binding to
ACE-2 receptors in type II pneumocytes in the lungs lead to local tissue destruction and initiation of an inflammatory cascade. ${ }^{6}$ Viral pathogen associated protein (PAMP) and tissue destruction released protein (DAMP) trigger off release of proinflammatory cytokines including interleukins IL-1B and IL-6, systemically causing sepsis syndrome. ${ }^{7}$

COVID-19 commonly presents as a mild upper respiratory illness with fever, cough, and sore throat. Less common symptoms include diarrhea, vomiting, change in sense of taste and smell, etc. In about $10 \%$ of patients, COVID-19 infection can progress into more severe illness needing hospitalization for pneumonia, ARDS, and sepsis secondary to cytokine storm. ${ }^{8}$ Some of these patients may develop cardiac injury, thrombo-embolism, kidney failure, and secondary infections. More recent studies suggest that many infected individuals, especially children and young adults, may remain asymptomatic. These asymptomatic individuals and many patients who are in the pre-symptomatic phase of

\footnotetext{
${ }^{*}$ Correspondence Author:

Janak Koirala, MD, MPH, FACP, FIDSA

Professor Emeritus,

Department of Medicine,

Division of Infectious Diseases

Southern Illinois University School of Medicine,

Springfield, Illinois, USA

Email: jkoirala@siumed.edu
} 
the illness may be playing a key role in spreading the virus. ${ }^{9}$

Transmission of SARS CoV-2 occurs mainly as a droplet infection directly through respiratory route or via direct contact with contaminated hands or fomites or surfaces where it has been found to be viable for a prolonged duration. However, it has also been found to be in faeces and in aerosolized form which are possibly additional but less common modes of transmission. The reproduction number (R0) of COVID-19 has been estimated to be 2.2 to 5.7 in various studies done in different countries..$^{10-12}$ Using time dependent (TD) method, the $\mathrm{R}(\mathrm{t})$ values for March 9, 2020 for European countries were 3.1 for Italy, 6.6 for France, 4.4 for Germany, and 3.9 for Spain. ${ }^{13}$ Based on these data other countries such as Singapore advocated social distancing to flatten the curve and to reduce R0 to 1.5 and this strategy has been implemented successfully in many countries. ${ }^{14}$

\section{COVID-19 in Nepal}

A 32-year-old Nepali man returning from Wuhan on January 9, 2020 was the first SARS CoV-2 positive case in Nepal. ${ }^{15}$ He presented at the outpatient department of the Sukraraj Tropical and Infectious Disease Hospital, Kathmandu on January 13, 2020- ten days after the start of symptoms. He was discharged home on January 17 , 2020 with instructions on strict home quarantine. His follow up RTPCR results done 12 days after discharge were negative for 2019nCov.

In order to prevent entry of the virus into the nation, on March 12th, Nepal government halted entry visas from countries that had community spread, viz. China, Italy, Spain, Iran, South Korea, Germany, France, and Japan. Travel restrictions were further expanded on March 14th. All entry visas and international flights were suspended, and all land entry posts shut down. Individuals entering the country were requested to stay in self-quarantine for 14 days. Other actions that were taken included monitoring temperature and health screening at 43 entry points via land from neighboring nations India and China, risk awareness campaigns, preventive measures (social distancing and masks), designation of several hospitals in all 7 provinces as COVID hospitals, creation of testing and management guidelines, and outlining case definition.

Additionally, facility for testing, specifically PCR and RDT, was made available in various provinces. Hotspots of potential COVID-19 are being continuously identified. According to WHO recommendation that all confirmed cases should be isolated in health facilities to prevent transmission and provide adequate care, the decision was made (on March 20th) to test all entry from India since individuals are still crossing the open India-Nepal border despite restrictions. Persons under investigation (PUI) are quarantined in government facilities and positive cases isolated in designated hospitals. If any individual tests positive on the PCR, surveillance officers are deployed and extensive contact tracing executed to identify additional potential cases.

The nation has been shut down since March 24th, 2020. Schools and businesses are closed and all travel via air or ground restricted. Only essential services such as pharmacies and grocery stores are open for a fixed time each day. Lock-down and isolation/ quarantine have been strictly reinforced by security sector- police, border management, and correction officers.

The second case was identified just before the lock-down was initiated. A 19-year-old who had arrived in Kathmandu from France via Qatar presented to the Sukraraj Tropical and Infectious Disease Hospital, Kathmandu with some symptoms and tested positive for SARS CoV-2. She was discharged after a few days after subsequent tests demonstrated negative results. Since then, until this write-up, over 100 cases have tested positive by the RT-PCR method and have been isolated in various hospitals in different provinces, 22 of whom have been discharged after subsequent negative test results. None of the cases needed admission into the intensive care unit and there have been no deaths. As of 7th May 2020, the Ministry of Health and Population (MOHP) of Nepal reported performing over 14500 PCR tests and over 54000 RDTs, whereas 133 people are on isolation and over 17000 people are on quarantine.

\section{Recent Development}

At the time of writing this article, there are no proven vaccines or antiviral drugs available to treat COVID-19. Clinical trials of vaccines and antiviral treatment are in progress. Two RNA polymerase inhibitors, Remdesivir (Gilead, USA) and Favipiravir (Avigan, Toyama Chemical, Japan) appear to be effective in treatment of COVID-19. In a study of Remdesivir used on a compassionate basis for hospitalized patients with severe COVID-19, 57\% patients receiving mechanical ventilation were extubated, $47 \%$ were discharged, and $13 \%$ died. ${ }^{16}$ Preliminary data released from NIH clinical trial showed that Remdesivir shortened median duration of illness from 15 days to 11 days; however, survival benefit was not significant. ${ }^{17}$ Based on these findings, US FDA (Food and Drug Administration) gave permission for its use in patients with severe COVID-19 under Emergency Use Authorization (EUA). An open-label trial in China showed patients receiving oral favipiravir plus inhaled interferon had a median viral shedding of 4 days compared to 11 days in a historical cohort of patients receiving lopinavir/ritonavir. ${ }^{18}$ An Indian pharmaceutical company has received permission for manufacturing generic version of favipiravir in India.

Convalescent plasma derived from recently recovered donors with high neutralizing antibody titers has been shown to improve the clinical outcomes in severe COVID-19 cases. ${ }^{19-20}$ Based on these studies and recommendations from authorities including $\mathrm{WHO}$, FDA and European Commission, Convalescent Plasma Therapy (CPT) is currently being investigated as a treatment option for patients with severe or immediately life-threatening COVID-19 infection. A prospective study for CPT is in the process of being approved by the Ministry of Public Health and Populations (MOHP) and Nepal Health Research Council (NHRC) so that it can be made available for severely ill patients in Nepal.

As noted above, pathogenesis of COVID-19 involves release of proinflammatory cytokines including interleukins-6 (IL-6). Based on results from retrospective studies that showed reduction in mortality in IL-6 inhibitor tocilizumab treated COVID-19 patients, IL-6 inhibitors such as sarilumab, siltuximab, tocilizumab are undergoing further study. ${ }^{8,21}$

\section{Conclusions}

Despite all the advances made in epidemiological investigation, diagnostic testing, and treatment modalities, COVID-19 has not only been the cause of high worldwide morbidity and mortality, but it has also created crises in economic, social, and psychological health of the countries around the globe which will take a longtime to recover. Although timely actions taken by Nepal has slowed down the spread of the virus, the number of cases is creeping up. Given an ongoing larger scale epidemic south of the border, spread of this epidemic in Nepal appears to be inevitable. It is important for Nepal to continue its preventive strategies of social distancing, active surveillance, and quarantine/isolation to slow down transmission of virus. Similarly, Nepal needs to intensify the preparations of healthcare institutions for treatment of infected individuals before the system gets overwhelmed.

Following are the important issues which need to be addressed in 
the coming days, weeks, months and maybe years:

1. Preparation to handle surge in COVID-19 cases- As Nepal has crossed its 100th case mark and the country is entering into the third phase of the epidemic, are we prepared to handle the surge of COVID-19 cases?

2. Preparing more manpower- As we have seen during this crisis, Nepal needs more trained manpower to run laboratories, intensive care units, ICU specialists, respiratory therapy, and infectious disease specialists among others.

3. Social distancing and lockdowns- how much social distancing is ideal? How long will the global society and world economy sustain social distancing and lockdowns?

4. Prevention by herd immunity- will there be an effective vaccine or will we have to gain natural herd immunity by exposing a large proportion of global population?

5. Preparation for resurgence- will there be a resurgence of the virus causing additional epidemics or pandemic next fall or winter? Will the countries around the world including Nepal be prepared for this resurgence?

6. Stronger epidemiological unit- Nepal needs a center with more authority and more trained manpower to face future epidemics, be it a similar respiratory virus or an entirely new pathogen. The country's epidemiological unit also needs the capacity to run a well-equipped molecular lab for molecular epidemiological studies in the country.

7. Strengthening research in Nepal- As we have observed during this crisis, there are opportunities for research, but Nepal lacks infrastructure and support system for researchers. This situation can be improved with the help of academic centers and NHRC partnership.

Disclosures: The authors do not have any conflict of interest with the content of this manuscript.

\section{References}

1. Rothan HA, Byrareddy SN. The epidemiology and pathogenesis of coronavirus disease (COVID-19) outbreak. J Autoimmun. 2020 Feb 26:102433. doi:10.1016/j.jaut.2020.102433. [Epub ahead of print] Review. PubMed PMID: 32113704; PubMed Central PMCID: PMC7127067.

2. Lu H., Stratton C.W., Tang Y.W. Outbreak of pneumonia of unknown etiology in wuhan China: the mystery and the miracle. J. Med. Virol. 2020;92(4):401-402.

3. Velavan TP, Meyer CG. The COVID-19 epidemic. Trop Med Int Health. 2020 Mar;25(3):278-280. doi: 10.1111/tmi.13383. Epub 2020 Feb 16. PubMed PMID:32052514.

4. Zhou P, Yang $X L$, Wang $X G$, et al. A pneumonia outbreak associated with a new coronavirus of probable bat origin. Nature. 2020 Mar;579(7798):270-273.

5. Andersen, K.G., Rambaut, A., Lipkin, W.I. et al. The proximal origin of SARS-CoV-2. Nat Med April 2020; 26:450-452.

6. Rabi FA, Al Zoubi MS, Kasasbeh GA, Salameh DM, Al-Nasser AD. SARS-CoV-2 and Coronavirus Disease 2019: What We Know So Far. Pathogens. 2020;9(3):231.

7. Vardhana SA, Wolchok JD. The many faces of the anti-COVID immune response. J Exp Med. 2020;217(6):e20200678.

8. Adarsh Bhimraj, Rebecca L. Morgan, et al. Infectious Diseases Society of America Guidelines on the Treatment and Management of Patients with COVID-19 Infection. Available online at: www.idsociety.org/COVID19guidelines (Last updated April 21, 2020). Accessed on: 6.5.2020.
9. Arons MM, Hatfield KM, Reddy SC, et al. Presymptomatic SARS-CoV-2 Infections and Transmission in a Skilled Nursing Facility [published online ahead of print, 2020 Apr 24]. N Engl J Med. 2020;10.

10. Riou J, Althaus CL. Pattern of early human-to-human transmission of Wuhan 2019 novel coronavirus (2019-nCoV), December 2019 to January 2020. Euro Surveill. 2020 Jan;25(4). doi: 10.2807/1560-7917.ES.2020.25.4.2000058. PubMed PMID: 32019669.

11. Chen J. Pathogenicity and transmissibility of 2019-nCoV-A quick overview and comparison with other emerging viruses. Microbes Infect. 2020 Mar;22(2):69-71. Epub 2020 Feb 4. PubMed PMID: 32032682; PubMed Central PMCID: PMC7102641.

12. Sanche S, Lin YT, Xu C, Romero-Severson E, Hengartner N, Ke R. High Contagiousness and Rapid Spread of Severe Acute Respiratory Syndrome Coronavirus 2. Emerg Infect Dis. 2020 Apr 7;26(7). doi: 10.3201/eid2607.200282. [Epub ahead of print] PubMed PMID: 32255761.

13. Yuan J, Li M, Lv G, Lu ZK. Monitoring Transmissibility and Mortality of COVID-19 in Europe. Int J Infect Dis. 2020 Mar 28. pii: S1201-9712(20)30182-X. doi: 10.1016/j.ijid.2020.03.050. [Epub ahead of print] PubMed PMID: 32234343.

14. Koo JR, Cook AR, Park M, Sun Y, Sun H, Lim JT, Tam C, Dickens BL. Interventions to mitigate early spread of SARS-CoV-2 in Singapore: a modelling study. Lancet Infect Dis. 2020 Mar 23. pii: S1473-3099(20)30162-6. doi:10.1016/S14733099(20)30162-6. [Epub ahead of print] PubMed PMID: 32213332.

15. Bastola A, Sah R, Rodriguez-Morales AJ, et al. The first 2019 novel coronavirus case in Nepal. The Lancet Infect Dis 2020. https://doi.org/10.1016/S1473-3099(20)30067-0

16. Grein J, Ohmagari N, Shin D, et al. Compassionate Use of Remdesivir for Patients with Severe Covid-19 [published online ahead of print, 2020 Apr 10]. N Engl J Med. 2020.

17. NIH clinical trial shows Remdesivir accelerates recovery from advanced COVID-19. NIH News Release, April 29, 2020. Available at: https://www.nih.gov/news-events/newsreleases/nih-clinical-trial-shows-remdesivir-acceleratesrecovery-advanced-covid-19

18. Favipiravir: A Potential Antiviral for COVID-19? NEJM Journal Watch, 9 April 2020.

19. Kai Duan, et al. Effectiveness of convalescent plasma therapy in severe COVID-19 patients. Proceedings of the National Academy of Sciences Apr 2020, 117 (17) 9490-9496.

20. Shen C, Wang Z, Zhao F, et al. Treatment of 5 Critically III Patients With COVID-19 With Convalescent Plasma [published online ahead of print, 2020 Mar 27]. JAMA. 2020;323(16):1582-1589.

21. NIH (National Institute of Health) COVID-19 Treatment Guidelines. Available at: https://www. covid19treatmentguidelines.nih.gov 